\title{
Some partition and analytical identities arising from the Alladi, Andrews, Gordon bijection
}

\author{
S. Capparelli ${ }^{1}$ A. Del Fra ${ }^{1} \cdot$ P. Mercuri ${ }^{2} \cdot$ A. Vietri $^{1}$ \\ Received: 6 April 2020 / Accepted: 19 August 2020 / Published online: 4 November 2020 \\ (c) The Author(s) 2020
}

\begin{abstract}
In the work of Alladi et al. (J Algebra 174:636-658, 1995) the authors provided a generalization of the two Capparelli identities involving certain classes of integer partitions. Inspired by that contribution, in particular as regards the general setting and the tools the authors employed, we obtain new partition identities by identifying further sets of partitions that can be explicitly put into a one-to-one correspondence by the method described in the 1995 paper. As a further result, although of a different nature, we obtain an analytical identity of Rogers-Ramanujan type, involving generating functions, for a class of partition identities already found in that paper and that generalize the first Capparelli identity and include it as a particular case. To achieve this, we apply the same strategy as Kanade and Russell did in a recent paper. This method relies on the use of jagged partitions that can be seen as a more general kind of integer partitions.
\end{abstract}

Keywords Partition identity · Rogers-Ramanujan identity · Jagged partition · Analytical identity

Mathematics Subject Classification Primary 11P84; Secondary 05A17 - 11P82 . 11P83

\section{Introduction}

In a 1969 paper, [2], Andrews characterized the type of partition sets that could be set into a bijection using Euler's classical trick to show that partitions of $n$ into distinct

P. Mercuri is supported by the research grant "Ing. Giorgio Schirillo" of the Istituto Nazionale di Alta Matematica "F. Severi", Rome.

\footnotetext{
$凶 \quad$ A. Vietri

andrea.vietri@uniroma1.it

1 Università degli Studi di Roma La Sapienza, Rome, Italy

2 Università degli Studi di Roma "Tor Vergata”, Rome, Italy
} 
parts are as many as partitions of $n$ into odd parts. In particular, Andrews proved that an identity of Schur ([12]) and one of Göllnitz ([10]) provide examples of "Euler-pairs". Inspired by that paper, here we look at one of the identities given in [6], see also [8]. We study the bijection provided by Alladi et al. in [1] and we find new sets of partitions that can be set into a bijection using the same approach. For further details on this subject and some generalizations we refer the reader to [3-5,7,9].

As the starting point of our research, we consider the partition identity which was proved in [1] and in particular the Concluding Remarks (Section 7), according to which the first Capparelli's identity (see [7]) can be generalized from modulo 3 to modulo $t$ by means of suitable dilations. In Sect. 2 we find an analytical identity for the partition identity modulo $t$. This is done using the same method as in [11] to compute the generating functions of the sum side. In Sect. 3 we again look back at [1], this time by generalizing the machinery which led the authors to build up the partition identity. In particular, we study a different class of partition identities which are indexed by two coprime integers $s$ and $t$. As better clarified in Sect. 3, these two parameters play different roles and actually generalize the roles of $s=2$ and $t=3$ in [1]. In the present setting we obtain a new family of partition identities.

\section{An analytical identity for an Alladi-Andrews-Gordon bijection}

Let $n, t$ be positive integers with $t>2$ and denote by $\mathcal{C}(n)_{t}$ the set of partitions of $n$ with distinct parts that are either divisible by $t$ or congruent to $t \pm 1(\bmod 2 t)$. Furthermore, let $\mathcal{D}(n)_{t}$ denote the set of partitions of $n$ with distinct parts larger than 1 that are either divisible by $t$ or congruent to $\pm 1(\bmod t)$ and whose difference is at least $t+1$, with the following exception: the difference between two adjacent parts can be smaller than $t+1$ if they are both divisible by $t$ or their sum is divisible by $2 t$. Alladi et al. proved the following proposition (see [1]).

Proposition $1 \mathcal{C}(n)_{t}$ and $\mathcal{D}(n)_{t}$ have the same cardinality.

In the present section we find an analytical expression for the above partition identity. The proof of our result is inspired by the argument in [11]. In accordance with that paper, we provide the following definition.

Definition 2 For a fixed positive integer $k$, a $k$-jagged partition is a finite sequence $\left(a_{1}, \ldots, a_{m}\right)$ such that $a_{1}, \ldots, a_{m} \in \mathbb{Z}, a_{1}>0$, and $a_{i+1}-a_{i} \geq-k$, for every $i=1, \ldots, m-1$.

Clearly, if $k=0$, we obtain the classical partitions written in weakly increasing order, as in [11]. The set of classical partitions $\left(b_{1}, \ldots, b_{m}\right)$ is easily seen to be in bijection with the set of $k$-jagged partitions $\left(a_{1}, \ldots, a_{m}\right)$ by associating $b_{i}$ to $a_{i}+(i-1) k$; essentially, we add a $k$-staircase. In the present paper, this bijection plays an important role when passing to generating functions. For our purposes, we now consider a special class of $k$-jagged partitions.

Definition 3 A $k$-jagged partition is called strong if it satisfies the condition $a_{i^{\prime}}-a_{i} \geq$ $-k$, for every $i=1, \ldots, m$ and $i^{\prime}=i+1, \ldots, m$. 
With this definition it is now possible to introduce the key concept we require for our proof.

Definition 4 For each positive integer $j$ and strong $k$-jagged partition $\mu$, we define the maximal block $\mathcal{M}_{j}$ corresponding to $j$ in the following way. If there is an element $a_{i}$ of $\mu$ such that $a_{i}=j$ and with the property that every element before $a_{i}$ is smaller than $j$, then $\mathcal{M}_{j}$ is the maximal subsequence of $\mu$ starting with $a_{i}$ whose elements belong to the set $\{j, j-1, j-2, \ldots, j-k\}$. If there is no element $a_{i}$ satisfying the above conditions, then $\mathcal{M}_{j}$ is the empty set.

The maximal blocks are in bijection with the positive integers and it is not hard to see that a given strong $k$-jagged partition is exactly the juxtaposition of all its maximal blocks. While it seems difficult to work out the general form of a maximal block, in our context such blocks enjoy some additional properties which make their description easy in order to obtain Theorem 5.

Example We have that

$$
\mu=(3,5,5,4,5,6,4,3,4,0,-2,5,11)
$$

is a 4-jagged partition and it is not a strong 4-jagged partition. However it is a 8-jagged partition and a strong 8-jagged partition. If we regard $\mu$ as a strong 8 -jagged partition, we have the following maximal blocks:

$$
\begin{aligned}
\mathcal{M}_{3} & =(3) \\
\mathcal{M}_{5} & =(5,5,4,5) \\
\mathcal{M}_{6} & =(6,4,3,4,0,-2,5) \\
\mathcal{M}_{11} & =(11), \\
\mathcal{M}_{j} & =\varnothing, \quad \text { for } j=1,2,4,7,8,9,10 \text { and } j \geq 12 .
\end{aligned}
$$

Adding the 8-staircase $(0,8,16,24,32,40,48,56,64,72,80,88,96)$ we get the classical partition $(3,13,21,28,37,46,52,59,68,72,78,93,107)$.

In the proof of the following theorem we need some further notation. Let $b$ be a finite subsequence; by $b^{*}$ we mean a string of either 0 or more contiguous blocks of $b$, and by $b^{\bullet}$ we mean either the empty string or $b$ itself. For instance, the notation $(6,4,6,3)^{*}(6,5)^{\bullet}$ is compatible with any of the following: $(6,5)$, $(6,4,6,3),(6,4,6,3,6,4,6,3,6,4,6,3)$, or $(6,4,6,3,6,4,6,3,6,5)$, but not with $(6,4,6,3,6,5,6,5)$ or $(6,4,6,3,6,5,6,4,6,3)$.

Theorem 5 Let $t$ be an integer greater than 2 and

$$
Q(a, b, c, d)=2 a^{2}+\frac{1}{2} b^{2}+c^{2}+d^{2}+2 a b+2 a c+2 a d+b c+b d+c d .
$$

We have

$$
\prod_{n \equiv 0, t-1, t, t+1(\bmod 2 t)}\left(1+q^{n}\right)=\sum_{a=0}^{\infty} \sum_{b=0}^{\infty} \sum_{c=0}^{\infty} \sum_{d=0}^{\infty} \frac{q^{t Q(a, b, c, d)+\frac{t}{2} b-c+d}}{\left(q^{2 t}, q^{2 t}\right)_{a}\left(q^{t}, q^{t}\right)_{b}\left(q^{t}, q^{t}\right)_{c}\left(q^{t}, q^{t}\right)_{d}},
$$


where the left-hand side is the generating function of the partitions with distinct parts congruent to $0, t-1, t, t+1(\bmod 2 t)$, and the right-hand side is the generating function of partitions with distinct parts greater than 1 and congruent to $0, \pm 1(\bmod t)$ such that the difference between consecutive parts is at least $t+1$ unless they are both $0(\bmod t)$ or their sum is $0(\bmod 2 t)$.

Proof It is straightforward to see that the infinite product is the generating function of $\mathcal{C}(n)_{t}$. With Proposition 1 in mind, it suffices to show that the quadruple sum is the generating function of $\mathcal{D}(n)_{t}$. For every positive integer $j$, the configurations that are not allowed in $\mathcal{D}(n)_{t}$ are the following:

- $j, j$;

- $t j-1, t j$

- $t j-1, t j+t-1$;

- $t j, t j+1$;

- $t j, t j+t-1$;

- $t j+1, t j+t-1$;

- $t j+1, t j+t$;

- $t j+1, t j+t+1$.

If we subtract a $t$-staircase, we get

- $j, j-t$;

- $t j-1, t j-t$;

- $t j-1, t j-1$;

- $t j, t j-t+1$;

- $t j, t j-1$;

- $t j+1, t j-1$;

- $t j+1, t j$

- $t j+1, t j+1$.

Hence, the maximal blocks are the following:

- $\mathcal{M}_{t j-1}=(t j-1, t j-t+1)^{*}(t j-1)^{\bullet}$;

- $\mathcal{M}_{t j}=(t j)^{*}$

- $\mathcal{M}_{t j+1}=(t j+1)^{\bullet}$.

It follows that the generating function is

$$
\begin{aligned}
& \prod_{j=1}^{\infty} \frac{1+x q^{t j-1}}{1-x q^{t j-1} \cdot x q^{t j-t+1}} \prod_{j=1}^{\infty} \frac{1}{1-x q^{t j}} \prod_{j=1}^{\infty}\left(1+x q^{t j+1}\right) \\
& \quad=\frac{\left(-x q^{t-1}, q^{t}\right)_{\infty}\left(-x q^{t+1}, q^{t}\right)_{\infty}}{\left(x^{2} q^{t}, q^{2 t}\right)_{\infty}\left(x q^{t}, q^{t}\right)_{\infty}} \\
& \quad=\sum_{a=0}^{\infty} \frac{x^{2 a} q^{t a}}{\left(q^{2 t}, q^{2 t}\right)_{a}} \sum_{b=0}^{\infty} \frac{x^{b} q^{t b}}{\left(q^{t}, q^{t}\right)_{b}} \sum_{c=0}^{\infty} \frac{x^{c} q^{(t-1) c} q^{\frac{t c(c-1)}{2}}}{\left(q^{t}, q^{t}\right)_{c}} \sum_{d=0}^{\infty} \frac{x^{d} q^{(t+1) d} q^{\frac{t d(d-1)}{2}}}{\left(q^{t}, q^{t}\right)_{d}} \\
& \quad=\sum_{a=0}^{\infty} \sum_{b=0}^{\infty} \sum_{c=0}^{\infty} \sum_{d=0}^{\infty} \frac{x^{2 a+b+c+d} q^{4 a+4 b+2 c^{2}+c+2 d^{2}+3 d}}{\left(q^{2 t}, q^{2 t}\right)_{a}\left(q^{t}, q^{t}\right)_{b}\left(q^{t}, q^{t}\right)_{c}\left(q^{t}, q^{t}\right)_{d}} .
\end{aligned}
$$


Now we add a $t$-staircase which, in terms of generating functions, corresponds to the substitution $x^{m} \mapsto x^{m} q^{\frac{t m(m-1)}{2}}$. Moreover, since we are not interested in taking account of the number of parts, we set $x=1$ and this gives the result.

Remark 6 For $t=3$ the sum of the previous proposition gives the same sum obtained in [11] using the substitution $x \mapsto 1, i \mapsto b, j \mapsto c, k \mapsto d, l \mapsto a$ and also the same sum obtained in [9, Equation (2.6)] using the substitution $r \mapsto d, s \mapsto c, t \mapsto$ $b, v \mapsto a, q \mapsto q^{3}, a \mapsto q^{-2}, b \mapsto q^{-4}$.

\section{Partition identities for the "s-rate, $t$-stack" case}

As mentioned in the Introduction, in the present section we construct an original class of partition identities which are indexed by two coprime integers $s$ and $t$, with $t$ odd. Given a partition of a positive integer $n$ into distinct parts, we list its parts in decreasing order, as in [1]. Fix three positive integers $n, s, t$, with $s, t$ coprime and greater than 1 ; denote by $\mathcal{C}(n)_{s}^{t}$ the set of partitions of $n$ with distinct parts multiple of $s$ or multiple of $t$. Let $W=\{h s+k t: h, k \in \mathbb{N}\}$ and $U=\mathbb{N}-W$, where $\mathbb{N}$ is the set of nonnegative integers. Notice that $U$ is finite because its largest element is $(s-1)(t-1)-1$.

Now denote by $\mathcal{D}(n)_{s}^{t}$ the set of partitions of $n$ with distinct parts $d_{1}, \ldots, d_{m}$, where the elements $d_{i_{1}}>d_{i_{2}}>\cdots>d_{i_{p}}$ are precisely those not congruent to $0(\bmod t)$, and with the following conditions which all parts $d_{i}$ must fulfill.

D0. Setting

$$
\begin{gathered}
f_{p}=d_{i_{p}}-\left(m-i_{p}\right) t, \\
f_{p-1}=d_{i_{p-1}}-\left(m-i_{p-1}-1\right) t, \\
\quad \vdots \\
f_{p-h}=d_{i_{p-h}}-\left(m-i_{p-h}-h\right) t, \\
\quad \vdots \\
f_{1}=d_{i_{1}}-\left(m-i_{1}-p+1\right) t,
\end{gathered}
$$

we require that $f_{p}$ be congruent to 0 or $t(\bmod s)$, and the same must hold for $f_{i}-f_{i+1}$, with $i=1, \ldots, p-1$.

D1. $d_{i} \in W$.

D2. If $d_{i} \equiv 0(\bmod t)$, then $d_{i}>t(m-i)$.

D3. If $d_{i}-d_{i+r}<t+1$ for some positive integer $r$, then at least one of the following conditions must be satisfied:

I. $d_{i}-d_{i+r} \not \equiv 0(\bmod s)$ and $d_{i} \equiv d_{i+r} \equiv 0(\bmod t)$;

II. $d_{i}-d_{i+r}=s j$ and $d_{i}+d_{i+r} \not \equiv \pm s j(\bmod s t)$.

Notice that $\mathcal{C}(n)_{2}^{t}$ has a different meaning from $\mathcal{C}(n)_{t}$; a similar remark concerns $\mathcal{D}(n)_{2}^{t}$ and $\mathcal{D}(n)_{t}$. We are going to prove the following result.

Proposition $7 \mathcal{C}(n)_{s}^{t}$ and $\mathcal{D}(n)_{s}^{t}$ have the same cardinality. 
In order to establish the above proposition we prove a stronger result, namely, Proposition 8 , for which some more terminology is needed. We denote by $\mathcal{C}\left(n ; i_{1}, \ldots, i_{t-1}, k\right)_{s}^{t}$ the subset of $\mathcal{C}(n)_{s}^{t}$ whose parts are grouped according to their congruence class as follows: $i_{1}$ parts congruent to $s(\bmod s t), \ldots, i_{t-1}$ parts congruent to $(t-1) s$ $(\bmod s t), k$ parts larger than $t\left(\sum_{j=1}^{t-1} i_{j}\right)$ and congruent to $0(\bmod t)$. Similarly, set $\mathcal{D}\left(n ; i_{1}, \ldots, i_{t-1}, k\right)_{s}^{t}$ to be the subset of $\mathcal{D}(n)_{s}^{t}$ having $i_{h}$ parts congruent to $h s$ $(\bmod t)$, with $1 \leq h \leq t-1$, and $k$ further parts congruent to $0(\bmod t)$. Now we proceed with the proof of the stronger result.

Proposition $8 \mathcal{C}\left(n ; i_{1}, \ldots, i_{t-1}, k\right)_{s}^{t}$ and $\mathcal{D}\left(n ; i_{1}, \ldots, i_{t-1}, k\right)_{s}^{t}$ have the same cardinality.

Proof Given a partition in $\mathcal{C}\left(n ; i_{1}, \ldots, i_{t-1}, k\right)_{s}^{t}$, we associate to it a partition in $\mathcal{D}\left(n ; i_{1}, \ldots, i_{t-1}, k\right)_{s}^{t}$, with an algorithm that generalizes the classical case $t=3$ in [1]; also our terminology traces back to that paper. Later we show that such a procedure is reversible.

Let $\pi \in \mathcal{C}\left(n ; i_{1}, \ldots, i_{t-1}, k\right)_{s}^{t}$.

Step 1 Setting $p=\sum_{j=1}^{t-1} i_{j}$, split $\pi$ into the subpartition $\pi_{1}=\left(a_{1}, \ldots, a_{p}\right)$ of those elements not congruent to $0(\bmod t)$ and the subpartition $\pi_{2}$ made up of those elements congruent to $0(\bmod t)$. Notice that two elements in $\pi_{1}$ have difference $s j$ only if they have sum not congruent to $\pm s j(\bmod s t)$, i.e., they satisfy D3-II. Indeed, suppose we have two parts $s \alpha, s \beta \in \pi_{1}$ such that $s \alpha-s \beta=s j$ and $s j$. Assuming, by contradiction, that $s \alpha+s \beta \equiv \pm s j(\bmod s t)$, since $\alpha=\beta+j$ we have either $2 s \beta+s j \equiv s j(\bmod s t)$ or $2 s \beta+s j \equiv-s j(\bmod s t)$. In the first case $2 \beta \equiv 0$ $(\bmod t)$. In the second, likewise, we have $2 \alpha \equiv 0(\bmod t)$. Since $t$ is odd, it follows that $\alpha$ or $\beta$ are congruent to $0(\bmod t)$, in both cases contradicting the assumption. Step 2 Split $\pi_{2}$ into the subpartitions $\pi_{5}$ and $\pi_{4}$ consisting, respectively, of those elements larger than $t p$ and those not greater. Set $\pi_{5}=\left(b_{1}^{\prime}, \ldots, b_{k}^{\prime}\right)$ and $\pi_{4}=$ $\left(b_{1}^{\prime \prime}, \ldots, b_{r}^{\prime \prime}\right)$.

Step 3 We construct the $t$-fold conjugate of $\pi_{4}$, in symbols $\pi_{4}^{*}$. If $\pi_{4}=\varnothing$, then $\pi_{4}^{*}=\varnothing$. Otherwise, set $b_{1}^{\prime \prime}=u_{1} t, b_{2}^{\prime \prime}=u_{2} t, \ldots, b_{r}^{\prime \prime}=u_{r} t$, with $u_{1} \geq u_{2} \geq \ldots \geq u_{r}$; then $\pi_{4}^{*}$ is the partition whose diagram has $t r$ columns. Specifically, consider $u_{r}$ rows with cardinality $t r, u_{r-1}-u_{r}$ rows with cardinality $t(r-1), \ldots, u_{2}-u_{3}$ rows with cardinality $2 t, u_{1}-u_{2}$ rows with cardinality $t$. Notice that the columns, taken as blocks of $t$ columns each, give the elements of $\pi_{4}$.

For example, for $t=7$, if $\pi_{4}=(35,14,7)$, divide by 7 each part thus obtaining $(5,2,1)$, and then form a diagram by using "blocks" of 7 squares

by stacking, respectively, 5 blocks, 2 blocks, 1 block, as follows:

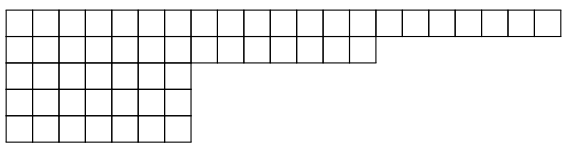

thus getting $\pi_{4}^{*}=(21,14,7,7,7)$.

Step 4 Let $\alpha_{1} \geq \alpha_{2} \geq \cdots \geq \alpha_{u_{1}}$ where $\alpha_{i}$ is the cardinality of the $i$ th row of the diagram associated to $\pi_{4}^{*}$. Add the partitions $\pi_{1}$ and $\pi_{4}^{*}$ by adding the corresponding 
parts $a_{i}+\alpha_{i}$ for $1 \leq i \leq u_{1}$ and leaving alone the elements $a_{u_{1}+1} \ldots a_{p}$. Notice that this is possible as $u_{1} \leq p$, since $b_{1}^{\prime \prime}=u_{1} t \leq t p$. We thus get a new partition $\pi_{6}=\left(a_{1}^{\prime}, \ldots, a_{p}^{\prime}\right)$, with $a_{i}^{\prime}>a_{i+1}^{\prime}$, for $i=1, \ldots, p-1$. This operation either leaves the differences between the parts unchanged or increases them by multiples of $t$ so that condition D3-II still holds. Moreover the condition D0 for the symbols $a_{i}^{\prime}$ in place of $f_{i}$ holds; in fact the difference between $a_{i}^{\prime}$ and $a_{i+1}^{\prime}$ can be either 0 or $t(\bmod s)$ and no larger multiples are possible, because of the structure of the diagram.

Step 5 Construct a string $\pi_{5} / \pi_{6}$ by juxtaposing, left to right, first the elements of $\pi_{5}$, then those of $\pi_{6}$. In this string $i_{h}$ parts are congruent to $h s(\bmod t)$, for $h=$ $1, \ldots, t-1$, and $k$ parts congruent to $0(\bmod t)$, the latter being larger than $t p$.

Step 6 Subtract multiples of $t$ to the elements of $\pi_{5} / \pi_{6}$, by obtaining the following new elements:

$$
\begin{aligned}
\bar{b}_{1}^{\prime} & =b_{1}^{\prime}-(p+k-1) t, \\
\bar{b}_{2}^{\prime} & =b_{2}^{\prime}-(p+k-2) t, \\
\vdots & \\
\bar{b}_{k}^{\prime} & =b_{k}^{\prime}-p t \\
\bar{a}_{1}^{\prime} & =a_{1}^{\prime}-(p-1) t, \\
\bar{a}_{2}^{\prime} & =a_{2}^{\prime}-(p-2) t, \\
\vdots & \\
\bar{a}_{p}^{\prime} & =a_{p}^{\prime}-0 t .
\end{aligned}
$$

Notice that while the elements $\bar{b}_{i}^{\prime}$ remain in a nonincreasing order, this does not necessarily happen for the elements $\bar{a}_{i}^{\prime}$. To be more precise, the nondecreasing order fails whenever $a_{i}^{\prime}-a_{i+1}^{\prime}=j<t$. In such a case $\bar{a}_{i+1}^{\prime}=\bar{a}_{i}^{\prime}+t-j>\bar{a}_{i}^{\prime}$. Moreover the elements $\bar{b}_{i}^{\prime}$ are all strictly positive since they are above the threshold value $t p$, while the elements $\bar{a}_{i}^{\prime}$ may be negative.

Step 7 Starting from the string $S_{0}=\left(\bar{b}_{1}^{\prime}, \ldots, \bar{b}_{k}^{\prime}, \bar{a}_{1}^{\prime}, \ldots, \bar{a}_{p}^{\prime}\right)$, we define a recursive algorithm which will lead us to a final string $S_{f}$ in $k$ steps. Define the generic $i$-th step, $1 \leq i \leq k$. Place $\bar{b}_{i}^{\prime}$ in the string $S_{i-1}$ in the rightmost possible position so that all the elements to its left are larger than itself. At the end of the process we get the desired string $S_{f}$.

Step 8 Denote by $c_{1}, \ldots, c_{p+k}$ the elements of $S_{f}$. We construct the following elements $d_{i}$, for $i=1, \ldots, p+k$ :

$$
\begin{aligned}
& d_{1}=c_{1}+(p+k-1) t \\
& d_{2}=c_{2}+(p+k-2) t, \\
& \vdots \\
& d_{p+k-1}=c_{p+k-1}+t, \\
& d_{p+k}=c_{p+k} .
\end{aligned}
$$


The difference $\Delta$ between two elements $d_{i}$ and $d_{i+1}$ that are not congruent to $0(\bmod t)$ may be less than $t+1$ only if $\Delta$ is a multiple of $s$ and the sum is not congruent to $\pm \Delta$ $(\bmod t)$.

Instead, if $d_{i} \not \equiv 0(\bmod t)$ and $d_{i+1} \equiv 0(\bmod t)\left(\right.$ or vice versa), we have $d_{i}=$ $c_{i}+(p+k-i) t$ and $d_{i+1}=c_{i+1}+(p+k-i-1) t$. Since $c_{i+1}-c_{i} \geq 1$, we deduce that $d_{i+1}-d_{i} \geq t+1$.

Notice that D0 holds. Indeed, the elements $f_{i}$ obtained from the elements $d_{i}$ not congruent to $0(\bmod t)$, by the formulas $(1)$, coincide with $a_{i}^{\prime}$ for which we already observed that they satisfy the requirements in Step 4. Therefore this algorithm transforms a partition $\pi$ in $\mathcal{C}\left(n ; i_{1}, \ldots, i_{t-1}, k\right)_{s}^{t}$ into a partition in $\mathcal{D}\left(n ; i_{1}, \ldots, i_{t-1}, k\right)_{s}^{t}$ that we denote by $\pi_{3}$ again in accordance with [1].

Finally, we only need to show that this procedure is completely reversible. Given a partition $\tilde{\pi}$ of $n$ in $\mathcal{D}\left(n ; i_{1}, \ldots, i_{t-1}, k\right)_{s}^{t}$ it is trivial to trace back the steps up to Step 5. We thus get a partition of $n$ made of some elements not congruent to 0 $(\bmod t)$ and of some elements congruent to $0(\bmod t)$. The set of the first type of elements, consistently with previous notation, we denote by $\pi_{6}=\left(a_{1}^{\prime}, \ldots, a_{p}^{\prime}\right)$, with $a_{i}^{\prime}>a_{i+1}^{\prime}$, for $i=1, \ldots, p-1$. The set of the second type of elements we denote by $\pi_{5}=\left(b_{1}^{\prime}, \ldots, b_{k}^{\prime}\right)$, with $b_{i}^{\prime}>b_{i+1}^{\prime}$, for $i=1, \ldots, k-1$.

Now, check whether $a_{p}^{\prime}$ is congruent to 0 or $t$ modulo $s$. In the first case do nothing. In the second case create a diagram with one row of $t$ squares. Proceed inductively by creating, in corresponding with each $a_{i}^{\prime}$, a row of squares to be stacked on top of the row corresponding to $a_{i+1}^{\prime}$, with the same number of squares of the row corresponding to $a_{i+1}^{\prime}$ if $a_{i}^{\prime} \equiv a_{i+1}^{\prime}(\bmod s)$; otherwise add $t$ new squares to the row. We thus form a diagram that gives, by using stacks of $t$ columns, the elements of $\pi_{4}$ and, using the rows (corresponding to $\pi_{4}^{*}$ ), the quantities to be subtracted from the elements of $\pi_{6}$, in order to obtain the elements of $\pi_{1}$. In this fashion, the partition $\pi$ in $\mathcal{C}\left(n, i_{1}, \ldots, i_{t-1}, k\right)_{s}^{t}$ is completely reconstructed.

Remark 9 If $t$ is even we can state an analogous proposition, but we must substitute the D3 condition with the following one:

$\mathrm{D}^{\prime}$. If $d_{i}-d_{i+r}<t+1$ for some positive integer $r$, then at least one of the following conditions must be satisfied:

I. $d_{i}-d_{i+r} \not \equiv 0(\bmod s)$ and $d_{i} \equiv d_{i+r} \equiv 0(\bmod t)$;

II. either at least one between $d_{i}$ and $d_{i+r}$ is equal to $s k \frac{t}{2}+h t$ for some nonnegative integers $h$ and $k$ or $d_{i}+d_{i+r} \not \equiv \pm s j(\bmod s t)$.

Remark 10 We observe that $\mathcal{D}(n)_{s}^{t}$ and $\mathcal{D}(n)_{t}^{s}$ have the same cardinality, because $\mathcal{C}(n)_{s}^{t}=\mathcal{C}(n)_{t}^{s}$.

We conclude this section with some remarks on the particular case $s=2$.

Remark 11 If $s=2$, the condition D0 is trivially satisfied.

Proposition 12 Condition D2 is redundant if $s=2$ and $t=3$.

Proof By contradiction, assuming that D2 does not hold, let $i_{0}$ be the largest integer such that $3 \mid d_{i_{0}} \leq 3\left(m-i_{0}\right)$ and let $d_{i_{0}+h}$ be the next multiple of 3 from left to right 
- if there is no such multiple, set $h=m-i_{0}+1$ and define $d_{m+1}=0$. Clearly, $d_{i_{0}+h} \geq 3\left(m-i_{0}-h\right)+3$. Notice that $h$ must be larger than 1 . The $h-1$ parts between $d_{i_{0}}$ and $d_{i_{0}+h}$ are all congruent to 1 or $2(\bmod 3)$. Now condition D3 forces the leftmost part to be not larger than $d_{i_{0}}-4$ and the rightmost to be not smaller than $d_{i_{0}+h}+4$. Furthermore, using condition D3, it is easy to see that every interval of the form $[\alpha, \alpha+5]$ contains at most 2 this $h-1$ parts. It follows that there are no more than

$$
2\left\lceil\frac{d_{i_{0}}-4-\left(d_{i_{0}+h}+4\right)+1}{6}\right\rceil
$$

parts of this kind. By hypotheses, if $h \leq m-i_{0}$ such a number does not exceed $2\left\lceil\frac{3 h-10}{6}\right\rceil$, which is less than $h-1$, a contradiction. In the remaining case, namely if $h=m-i_{0}+1$, the element $d_{m}$ might be equal to 2 but the above argument is still valid, using similar calculations, as long as $h$ is even. Instead, if $h$ is odd (necessarily $h \geq 3$ ), we slightly improve the argument. Since $d_{m-1} \geq 4$, counting the $h-2$ parts from $d_{i_{0}+1}$ to $d_{m-1}$ leads to the following contradiction:

$$
2\left\lceil\frac{d_{i_{0}}-4-4+1}{6}\right\rceil \leq 2\left\lceil\frac{3(h-1)-7}{6}\right\rceil \leq h-3 .
$$

Notice that for $t$ odd and larger than 3 Condition D2 is necessary.

\section{Examples}

In this section we give three examples of the bijection described in the previous section.

Example 13 We illustrate an example of the bijection when $s=2$ and $t=7$. Let $\pi=(84,70,66,46,40,38,35,14,10,8,7,4,2) \in \mathcal{C}(424)_{2}^{7}$.

Step 1 Split $\pi$ in

$$
\pi_{1}=(66,46,40,38,10,8,4,2), \quad \pi_{2}=(84,70,35,14,7) .
$$

So $p=8$ and the threshold is $t p=56$.

Step 2 Split $\pi_{2}$ in

$$
\pi_{5}=(84,70), \quad \pi_{4}=(35,14,7),
$$

and hence $k=2$ and $r=3$.

Step $3 \pi_{4}=(35,14,7) \mapsto(5,2,1)$

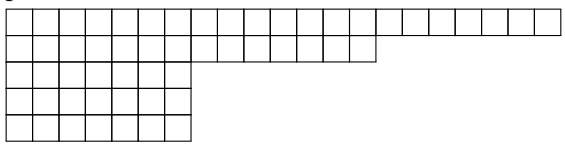

which gives the partition $\pi_{4}^{*}=(21,14,7,7,7)$. 
Step $4 \pi_{6}=\pi_{1}+\pi_{4}^{*}=(66+21,46+14,40+7,38+7,10+7,8,4,2)=$ $(87,60,47,45,17,8,4,2)$.

Step 5 Form the string $\pi_{5} / \pi_{6}=(84,70,87,60,47,45,17,8,4,2)$.

Step 6 Subtract from the string just obtained multiples of 7 as follows:

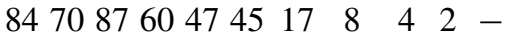

$$
\begin{aligned}
& 63564942352821 \quad 14 \quad 7 \quad 0=
\end{aligned}
$$

$$
211438181217-4-6-32
$$

Step 7 Starting from the string $S_{0}$ obtained in the previous step, move 14 as far right as possible thus obtaining

$$
S_{1}=(21,38,18,14,12,17,-4,-6,-3,2) .
$$

Next, move similarly the number 21 obtaining

$$
S_{2}=(38,21,18,14,12,17,-4,-6,-3,2)=S_{f}
$$

Step 8 Now we add again to $S_{f}$ the string of multiples of 7 as before

$$
\begin{array}{llllllllcccc}
38 & 21 & 18 & 14 & 12 & 17 & -4 & -6 & -3 & 2 & + \\
63 & 56 & 49 & 42 & 35 & 28 & 21 & 14 & 7 & 0 & = \\
101 & 77 & 67 & 56 & 47 & 45 & 17 & 8 & 4 & 2
\end{array}
$$

getting the partition $\pi_{3}=(101,77,67,56,47,45,17,8,4,2) \in \mathcal{D}(424)_{2}^{7}$ as desired.

We now check that this process is reversible. Given the partition $\pi_{3}=$ $(101,77,67,56,47,45,17,8,4,2)$, the steps $8,7,6,5$ are easily reversible and lead to $\pi_{5}$ and $\pi_{6}=(84,70,87,60,47,45,17,8,4,2)$. To recover $\pi_{4}$ proceed as follows. Note the position where, starting from the right, we find the first odd part. In our example it is 17 and, correspondingly, we draw a row of 7 squares. Going leftward and ignoring the multiple of 7 , we have the sequence $(17,45,47,60,87)$ that is congruent to $(1,1,1,0,1)$ modulo 2 . For each element with the same parity of the previous one, we draw a row upon the others with the same number of squares than the row below it. For each element with a different parity than the previous one, we draw a row upon the others with 7 squares more than the row below it. This generates the diagram

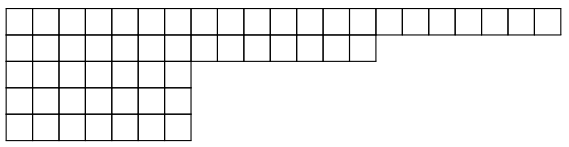

that coincides with the diagram in Step 3. In this diagram there are 3 blocks of 7 columns containing 35,14 and 7 squares, respectively. Hence $\pi_{4}=(35,14,7)$. The rows of the diagram give the string $(21,14,7,7,7)$ which must be subtracted to $(87,60,47,45,17)$ to get $\pi_{1}$. 
Example 14 We illustrate an example of the bijection when $s=3$ and $t=7$. Let $\pi=(91,84,75,63,54,49,39,36,21,18,14,9,3) \in \mathcal{C}(556)_{3}^{7}$. Step 1 Split $\pi$ in

$$
\pi_{1}=(75,54,39,36,18,9,3), \quad \pi_{2}=(91,84,63,49,21,14) .
$$

So $p=7$ and the threshold is $t p=49$.

Step 2 Split $\pi_{2}$ in

$$
\pi_{5}=(91,84,63), \quad \pi_{4}=(49,21,14)
$$

and hence $k=3$ and $r=3$.

Step $3 \pi_{4}=(49,21,14) \mapsto(7,3,2)$

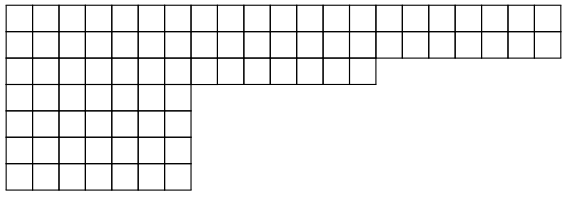

which gives the partition $\pi_{4}^{*}=(21,21,14,7,7,7,7)$.

Step $4 \pi_{6}=\pi_{1}+\pi_{4}^{*}=(75+21,54+21,39+14,36+7,18+7,9+7,3+7)=$ $(96,75,53,43,25,16,10)$.

Step 5 Form the string $\pi_{5} / \pi_{6}=(91,84,63,96,75,53,43,25,16,10)$.

Step 6 Subtract from the string just obtained multiples of 7 as follows:

$$
\begin{array}{ccccccccccc}
91 & 84 & 63 & 96 & 75 & 53 & 43 & 25 & 16 & 10 & - \\
63 & 56 & 49 & 42 & 35 & 28 & 21 & 14 & 7 & 0 & =
\end{array}
$$$$
2828145440252211910
$$

Step 7 Starting from the string $S_{0}$ obtained in the previous step, move the first 28 as far right as possible thus obtaining

$$
S_{1}=(28,14,54,40,28,25,22,11,9,10) .
$$

Moving the second 28, we obtain

$$
S_{2}=(14,54,40,28,28,25,22,11,9,10) .
$$

Finally, moving 14 we get

$$
S_{3}=(54,40,28,28,25,22,14,11,9,10)=S_{f}
$$


Step 8 Now we add again to $S_{f}$ the string of multiples of 7 as before

$5440282825221411910+$

$635649423528211470=$

117967770605035251610

getting the partition $\pi_{3}=(117,96,77,70,60,50,35,25,16,10) \in \mathcal{D}(556)_{3}^{7}$ as desired.

We now check that this process is reversible. Given the partition $\pi_{3}=$ $(117,96,77,70,60,50,35,25,16,10)$, the steps $8,7,6,5$ are easily reversible and lead to $\pi_{5}=(91,84,63)$ and $\pi_{6}=(96,75,53,43,25,16,10)$. To recover $\pi_{4}$ proceed as follows. Note the position where, starting from the right of $\pi_{6}$, we find the first element congruent to 7 (i.e., congruent to 1) modulo 3 . In our example it is 10 and, correspondingly, we draw a row of 7 squares. Going leftward, we have the sequence $(10,16,25,43,53,75,96)$ that is congruent to $(1,1,1,1,2,0,0)$ modulo 3 . For each element with the same congruence of the previous one mod 3 , we draw a row upon the others with the same number of squares than the row below it. For each element with a different congruence than the previous one, we draw a row upon the others with 7 squares more than the row below it. This generates the diagram

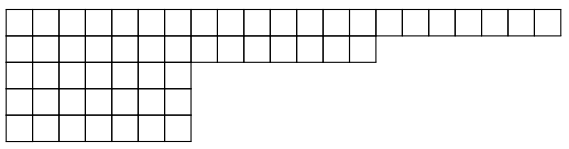

that coincides with the diagram in Step 3. In this diagram there are 3 blocks of 7 columns containing 49, 21 and 14 squares, respectively. Hence $\pi_{4}=(49,21,14)$. The rows of the diagram give the string $(21,21,14,7,7,7,7)$ which must be subtracted to $(96,75,53,43,25,16,10)$ to get $\pi_{1}$.

Example 15 Consider again $\pi=(91,84,75,63,54,49,39,36,21,18,14,9,3)$, but now as element in $\mathcal{C}(556)_{7}^{3}$. We show how to obtain the corresponding element of $\mathcal{D}(556)_{7}^{3}$.

Step 1 Now $\pi_{1}=(91,49,14)$ and $\pi_{2}=(84,75,63,54,39,36,21,18,9,3)$; hence $p=3$ and the threshold is $t p=9$.

Step 2 Split $\pi_{2}$ in $\pi_{5}=(84,75,63,54,39,36,21,18)$ and $\pi_{4}=(9,3)$.

Step $3 \pi_{4}=(9,3) \mapsto(3,1)$ and the diagram is

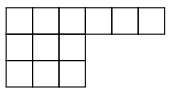

which gives the partition $\pi_{4}^{*}=(6,3,3)$.

Step $4 \pi_{6}=\pi_{1}+\pi_{4}^{*}=(91+6,49+3,14+3)=(97,52,17)$.

Step $5 \pi_{5} / \pi_{6}=(84,75,63,54,39,36,21,18,97,52,17)$. 
Step 6 Subtract from $\pi_{5} / \pi_{6}$ multiples of 3 as follows:

$$
\begin{array}{llllllllccc}
84 & 75 & 63 & 54 & 39 & 21 & 18 & 97 & 52 & 17 & - \\
27 & 24 & 21 & 18 & 15 & 12 & 9 & 6 & 3 & 0 & =
\end{array}
$$

$$
5751423624999914917
$$

Step 7 Starting from the string $S_{0}$ obtained in the previous step and moving the multiples of 3 by the recursive method described before, we get

$$
S_{f}=(91,57,51,49,42,36,24,17,9,9) .
$$

Step 8 Now we add again to $S_{f}$ the string of multiples of 3 as before

$915751494236241799+$

$27242118151296 \quad 6 \quad 30=$

11881726757483323129

getting the partition $\pi_{3}=(118,81,72,67,57,48,33,23,12,9) \in \mathcal{D}(556)_{7}^{3}$ as desired.

Also in this case it is easy to invert the process.

Acknowledgements The manuscript has been improved by following the suggestions of the anonymous referee, to whom we are grateful.

Funding Open access funding provided by Università degli Studi di Roma La Sapienza within the CRUICARE Agreement.

Open Access This article is licensed under a Creative Commons Attribution 4.0 International License, which permits use, sharing, adaptation, distribution and reproduction in any medium or format, as long as you give appropriate credit to the original author(s) and the source, provide a link to the Creative Commons licence, and indicate if changes were made. The images or other third party material in this article are included in the article's Creative Commons licence, unless indicated otherwise in a credit line to the material. If material is not included in the article's Creative Commons licence and your intended use is not permitted by statutory regulation or exceeds the permitted use, you will need to obtain permission directly from the copyright holder. To view a copy of this licence, visit http://creativecommons.org/licenses/by/4.0/.

\section{References}

1. Alladi, K., Andrews, G.E., Gordon, B.: Refinements and generalizations of Capparelli's conjecture on partitions. J. Algebra 174, 636-658 (1995)

2. Andrews, G.E.: Two theorems of Euler and a general partition theorem. Proc. Am. Math. Soc. 20, 499-502 (1969)

3. Andrews, G.E.: The Theory of Partitions. Cambridge University Press, Cambridge (1984)

4. Andrews, G.E.: Schur's Theorem, Capparelli's Conjecture and $q$-Trinomial Coefficients. Contemporary in Mathematics, vol. 166, American Mathematical Society, Providence (1994)

5. Capparelli, S.: Elements of the annihilating ideal of a standard module. J. Algebra 145, 32-54 (1992)

6. Capparelli, S.: On some representations of twisted affine Lie algebras and combinatorial identities. J. Algebra 154(2), 335-355 (1993) 
7. Capparelli, S.: A combinatorial proof of a partition identity related to the level 3 representations of a twisted affine Lie algebra. Commun. Algebra 23(8), 2959-2969 (1995)

8. Capparelli, S.: A construction of the level 3 modules for the affine lie algebra $A_{2}^{(2)}$ and a new combinatorial identity of the Rogers-Ramanujan type. Trans. Am. Math. Soc. 348(2), 481-501 (1996)

9. Dousse, J., Lovejoy, J.: Generalizations of Capparelli’s identity. Bull. Lond. Math. Soc. 51(2), 193-206 (2019)

10. Göllnitz, H.: Partitionen mit Differenzenbedingungen. J. Reine Angew. Math. 225, 154-190 (1967)

11. Kanade, S., Russell, M. C.: Staircases to analytic sum-sides for many new integer partition identities of Rogers-Ramanujan type. Electron. J. Combin. 26(1), Paper 1.6 (2019)

12. Schur, I.: Zur additiven Zahlentheorie, pp. 488-495. S.-B. Akad. Wiss. Berlin (1926)

Publisher's Note Springer Nature remains neutral with regard to jurisdictional claims in published maps and institutional affiliations. 